\title{
De la magie des sons à la magie du monde
}

\section{Christiane Dunoyer}

\section{OpenEdition}

\section{Journals}

Édition électronique

URL : http://journals.openedition.org/esp/1633

DOI : 10.4000/esp. 1633

ISSN : 2532-0319

\section{Éditeur}

Centre d'Information sur l'Éducation Bilingue et Plurilingue

\section{Édition imprimée}

Date de publication : 1 décembre 2017

Pagination : 77-81

ISSN : 1127-266X

\section{Référence électronique}

Christiane Dunoyer, « De la magie des sons à la magie du monde », Éducation et sociétés plurilingues

[En ligne], 43 | 2017, mis en ligne le 01 février 2019, consulté le 15 septembre 2020. URL : http:// journals.openedition.org/esp/1633 


\section{DE LA MAGIE DES SONS À LA MAGIE DU MONDE}

\section{Christiane DUNOYER}

Nata ad Aosta il 16 luglio 1972 da genitori valdostani, è cresciuta e vive ancora in questa regione in cui la variazione linguistica è un'esperienza quotidiana. Sensibile a tutto quanto è in rapporto con la comunicazione, condivide qui $i$ suoi ricordi rispetto alle numerose lingue con cui è venuta in contatto. Da antropologa, presenta uno sguardo originale sulla sua esperienza personale, ma nel corso del suo articolo tenta di fornire una testimonianza quanto più veritiera possibile.

Parole chiave: Francoprovenzale, patois, variazione, connivenza, emozione

Born in Aosta July 16, 1972 of Valdaostan parents, she grew up and still lives in this region, where linguistic variation is a day-to-day experience. Awake to everything that concerns communication, she shares her memories of the many languages with which she came into contact. As an anthropologist, she casts an objective eye upon her own life expérience, but tries to deliver, as she goes along, an as authentically personal account as possible.

Keywords: Francoprovençal, patois, variation, complicity, emotion

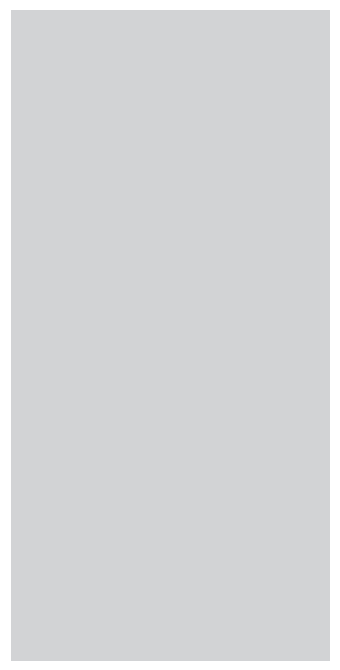

Y'est parce qu'il est question de moi que ce texte à autant tardé à venir au monde. C'est parce qu'il est question de mes habi$\lambda_{\text {tudes linguistiques, et dans les habitudes linguistiques il est }}$ quelque chose de très intime, de douloureux parfois. C'est parce que j'ai toujours accordé un rôle de premier plan aux mots au cours de ma vie, car il y a de la magie dans les sons qui se succèdent, au-delà du sens qui est souvent une évidence, dans le choix de la langue, de la connivence souhaitée, recherchée ou refusée.

Depuis ma première enfance, j'ai été exposée au francoprovençal, la langue de ma famille, au français et à l’italien. Je n'ai pas de souvenirs concernant mes expériences linguistiques, avant l'école maternelle. C'est à ce moment-là que les langues sont devenues une affaire sérieuse. Mon premier mot de français: j'avais trois ans, devant une diapo en noir et blanc: un enfant approche un verre de sa bouche «il boit». J'enregistre. D'autres mots en noir et blanc se sont ajoutés à celui-là: il fallait le faire, j'ai appris. Comme j'ai appris les ensembles, les coulcurs, les nombres, dans les polycopićs. J'étais docile, j’aimais 
De la magie des sons à la magie du monde C. DUNOYER le violet, mais ce n'était pas bien, il fallait se servir de tous les feutres. Et puis il y avait les diapos en noir et blanc et des mots qui n'étaient pas ceux que j'avais dû apprendre en entrant à l'école: dans les salles de classes et dans les salles de jeux tout parlait italien. Il avait suffi de le savoir, on avait parlé italien. On l'avait pris là où on l'avait trouvé, pas à la télé qui était encore trop rébarbative pour nous à l'époque (c'était en 1975!), mais au marché, pendant les visites du médecin, chez quelques voisins. Je dis on car l'individualité de l'interlocuteur venait en second plan à cet âge-là, d'après mes souvenirs, il y avait un sentiment diffus, ou confus, on se dispersait dans ces vastes espaces, on se dissipait dans le groupe. Et on se passait les mots dans les cours de récréation, autant qu'on s'en passait, car certains enfants en avaient beaucoup, ils n'avaient que ça comme monnaie d'échange, tandis que les autres se regardaient dans les yeux et se savaient de la même souche, on se guettait de loin, lorsque les mamans nous accompagnaient jusque devant le portail en susurrant des mots en patois (c'est ainsi que nous nommons le francoprovençal dans la vie quotidienne, sans aucune connotation négative, et c'est ainsi que je m'y réfêre dans ce texte lorsqu'il est question de mon point de vue intrinsèque) inquiètes qu'on apprenne bien l'italien. C'était leur souci. Nous, on levait les épaules, on se reconnaissait dans la même vibration, dans ce sanglot long qu'on ravalait à longueur de journée, à cause de cette coupure entre la vie palpitante là-dehors et la langue lisse des heures de classe, luisante comme les carrelages des couloirs, faite de mots intelligibles, mais vides pour le cœur.

Rapidement, au gré des diapos, «la pomme», «la table», etc. j’ai reconnu la langue de chez moi, c'était toujours en noir et blanc, mais ça caressait l'oreille. À la maison c'était souvent un peu plus en a, mais peu importe, «la pomma» n'est pas «la mela». Ça faisait du bien aussi quand on descendait des salles de classe pour se rendre à la cantine. Ce n'était pas le parfum de la cuisine de maman, mais on entendait parler patois, j'en avais les larmes aux yeux tellement j'étais envahie par la nostalgie de là-dehors. J'aurais voulu m'arrêter là-bas toute la journée, c'était si accueillant.

Un autre souvenir d'école maternelle: on chantait «Meunier tu dors...», c'était un peu comme les voix des cuisinières, un son qui venait de la maison, il était en couleurs, il portait la vie. Car à la maison on parlait le patois, mais le français était là dans les prières des grands-mères qui se terminaient par «ainsi soit-il», dans les noms de certains objets mystérieux «la cocotte», «la chicorée», «le duvet», «le bain-marie», «l'abat-jour», dans les conversations avec les membres de la famille émigrés en France et en Suisse, dans le vécu transfrontalier des aînés de la famille qui se traduisait par des liens concrets, par 
De la magie des sons à la magie du monde c. DunOYeR des départs et des retours impromptus, par des achats d'objets. Dans l'archéologie des valeurs familiales... Le français était surtout présent dans les chansons: j'aimais chanter et on chantait beaucoup. Et dans les livres de la bibliothèque familiale, qui incarnaient le prestige d'une culture: Jean-Baptiste De Tillier, le Coutumier...

Dans ma tête tout se combinait pour produire mon idée de «valdôtain», de chez nous, tant et si bien qu'il me faudra des années pour discerner l'élément francoprovençal de l'élément français. J'avais 22 ans le jour où un ami savoyard me demanda de lui envoyer des chansons valdôtaines en patois: je lui avais répondu qu'il y en avait beaucoup, mais il me fallut les chanter une à une pour trier celles qui étaient en francoprovençal et celles qui étaient en français, tellement elles m'appartenaient en profondeur, loin de toute appréhension rationnelle.

Quand le besoin me vint, je dis bien le besoin car c'est de cela qu'il s'agit, d'écrire mes sentiments profonds et d'inventer des histoires, tout naturellement je le fis en français, qui était ma langue écrite, étant donné que je n'avais jamais pensé au cours de ma jeunesse de pratiquer le francoprovençal à l'écrit, au-delà de quelques brèves phrases facétieuses. Ce furent des poèmes d'abord. Par la suite, j'ai publié deux romans. Un troisième est peut-être là, en train de sortir du flou de mes pensées tourbillonnantes. Il y a sept ans, après avoir lu mon dernier roman, un ami savoyard m'a encouragé à utiliser le francoprovençal, à trouver les mots à l'écrit, tout comme je saurais les trouver en parlant... En rentrant très tard ce soir-là je trouvai les mots et en trois mois j'écrivis dix petits poèmes! Il fallait que je me relâche, que j'oublie les discussions autour de la graphie et des formes pures... Qui parle la langue pure?

Depuis toujours, c'est-à-dire, déjà avant mes premiers souvenirs, deux patois cohabitaient dans mon moi profond: celui de mon père, d'Excenex, village de la colline d'Aoste, et celui de ma mère, de La Magdeleine. Je passais de l'un à l'autre avec bonheur mais avec des émotions différentes, car chaque façon de parler s'associait à des personnes et à des situations différentes. Et combien de fois par la suite j'ai redécouvert ces émotions grâce à la langue, à ses sonorités ou à ses expressions typiques. Alors, quand j'ai découvert que les patois n'étaient pas deux comme je l'avais supposé dans ma première enfance, mais très nombreux, j'ai commencé à apprécier toute cette gamme infinie de variations et à mes yeux le francoprovençal était un arc-en-ciel merveilleux qui abritait tout le monde, irradié chacun d'une lumière de couleur un peu différente. Je me souviens que je ressentais une émotion très forte à écouter les différences et assez rapidement il me parut naturel de tenter de les reproduire, car parler une 
certaine variété plutôt qu'une autre n'est pas un choix neutre: il suffit d'essayer une fois pour le lire dans les yeux de nos interlocuteurs. Plaisir relationnel mais aussi plaisir sensoriel, quand on fait rouler des mots nouveaux dans sa bouche, qu'on oblige sa propre langue à claquer là où elle n'a pas l'habitude et ses oreilles à écouter des suites de sons qui ne sont pas ceux que notre bouche prononçait jusqu'à la veille...

L'anglais ne me donnait pas ces émotions: au lycée, les méthodes d'enseignement avaient changé depuis la maternelle, ce n'était plus des diapos en noir et blanc, c'était le magnétophone grinçant avec haut-parleur et rallonge orange, dont le déroulement prenait cinq minutes à chaque fois. C'était encore des mots privés de la joie de la communication, la tristesse de la grammaire coupée de l'émotion. À part les vacances à la mer, les voyages étaient rares dans mon enfance. Je rêvais en regardant les cartes postales, en glanant des mots là où je les trouvais...Un jour sur une plage un garçon anglais m'offrit un coquillage: «shell», je n'oublierai jamais ce mot. Enfin un mot qui n'était pas vide. D'autres mots suivirent et donnèrent une vie à cette langue aussi. Je pouvais désormais composer des phrases sans passer par la traduction, je me réjouissais d'articuler des mots et de matérialiser mes pensées.

$\grave{\Lambda}$ chaque fois, il me fallait vivre une expérience de communication pour déclencher en moi la force vitale de la langue. Ça allait de plus en plus vite, au gré des rencontres, des lectures, des voyages... On me disait «tu peux voyager parce que tu connais les langues» je crois que l'inverse serait plus correct.

Un jour, je réalisais que le but n'était plus la langue mais la communication, le plaisir d'approcher des personnes et de mieux les comprendre à travers leur système. Je connus l'allemand ainsi. D'autres formes francoprovençales. L'occitan alpin. Des mots de basque. Le portugais. Certaines langues, je les parle couramment, d'autres je les comprends bien. D'autres j'aime en écouter les sonorités, je me fais bercer par ces locuteurs, sans leur avouer que je comprends moins que ce qu'ils croient, mais sans cette petite tricherie je serais privée du plaisir de les écouter: ils me parleraient une autre langue pour venir vers moi, comme les hauts-valaisans, dont je capte quelques mots par-ci par-là... J'aurais pu choisir l'allemand en langue 3 à l'université, quand j'étais en lettres modernes, mais je n'avais pas beaucoup de temps pour m'y consacrer, alors j'ai préféré une langue qui me permette de parler rapidement comme l'espagnol. Le castillan, comme disait mon premier professeur. Je me suis inscrite directement au niveau avancé, j’ai senti le défi que cette langue me lançait. La compréhension était là, presque totale dès le premier jour: le 
bonheur...et la hâte me prit de parler et d'écrire à mon tour. Je sentais le même élan que j'avais ressenti en passant d'une variété francoprovençale à une autre. Je plongeai dans la vaste littérature hispanique... Des relations professionnelles suivirent peu de temps après, des conversations téléphoniques (quand internet n'était pas encore un outil ordinaire) et même des interviews.

La chanson aussi m'a servi de pont, car elle nous expose à un riche vocabulaire. C'est ainsi que je peux affirmer avoir appris à comprendre le corse, mais avant encore ce système m'a permis de parler le patois d'Ayas. Les premières fois que je me lançais dans l'expérience, je prenais un ton facétieux et sortais des phrases parfaites quand le contexte s'y prêtait, en n'utilisant que les mots des chansons que je connaissais. Malgré ce soupçon de moquerie, qui d'habitude instille la bonne humeur auprès de mes interlocuteurs, cela resserrait les liens pendant la conversation et mes compétences augmentaient beaucoup plus rapidement.

Mais dans la communication, je n'ai pas fait que l'expérience de la proximité. À Aoste, quand je parle italien avec des personnes que je ne connais pas, on me demande souvent depuis combien de temps je suis dans la région, comme s'il ne faisait aucun doute que je viens d'ailleurs. D'où? De France, de Suisse, de Moldavie, de Pologne, du Canada, me dit-on... Mon accent? Le jeu des clichés? Je ne sais trop, mais de me sentir comme une étrangère chez moi, cela me vexe parfois. Mes fils aussi à l'école ont un peu souffert de la curiosité des adultes qui en faisait parfois des objets un peu rares, alors qu'ils étaient tellement à leur aise au milieu des langues parlées en famille et des langues introduites chez nous par le biais de filles et de garçons au pair que nous avons hébergés pendant des années. Pour quelqu'un, les langues restent un exercice intellectuel. Pour moi, c'est avant tout une ouverture sur un monde magique. En arrivant en Bretagne l'année dernière, vingt ans après mon dernier séjour, seulement en parcourant l'autoroute, je retrouvai des mots que je croyais oubliés. De vieux souvenirs jaillissaient de ma mémoire, des mots ensevelis revenaient à moi: les mots et les paysages en même temps! Jusqu'à rêver en breton une nuit, des mots que j'ai pu traduire le lendemain avec l'aide d'un garçon de café, en découvrant ainsi qu'ils avaient tout leur sens.

Et ce francoprovençal qui était au début de mon histoire, il est encore là, au cœur de ma vie, plus vivant que jamais. Je dirais langue charnelle, plutôt que langue maternelle (car c'est aussi ma langue paternelle, ma langue du berceau, même si j'ai perdu les souvenirs de moi bébé...). Car tous les jours je sens que cette langue m'appartient comme ma propre chair et s'enfonce au plus profond de moi-même 
pour exprimer tout ce qu'il y a de plus primordial et d'irréfléchi en moi. Les autres langues, quant à elles, se sont installées plus haut, dans la tête probablement: elles me suggèrent l'émotion de la découverte, l'ivresse de l'altérité, l'innovation dans mon existence, l'ouverture au monde... Les connaître et les pratiquer toutes, selon les circonstances, c'est un aller-retour infini, de moi au monde, entre introversion et extraversion. En même temps, j'ai des amis toujours plus nombreux avec lesquels partager la langue francoprovençale dans un espace toujours moins imaginaire à cheval sur trois pays, qui m'offre tous les jours une émotion nouvelle à travers ses mille formes que parfois j'écoute, que parfois je répète au gré des jeux subtils qui soustendent toute situation de communication, ses règles et ses enjeux, question tantôt de se rapprocher, tantôt de marquer une distance, tantôt de tendre la main à un locuteur hésitant, tantôt de céder à un plaisir purement esthétique. Parfois le son typique crée l'intimité, parfois la forme plus générale me conforte dans la complicité collective que je mets en place... En tant que directrice du Centre d'Etudes Francoprovençales, je plonge dans cette langue à tous les niveaux et je côtoie des esprits passionnés, des chercheurs rigoureux de plusieurs pays, des connaisseurs raffinés des subtilités de la langue. Tout en le parlant depuis toujours, j'ai compris la nécessité de développer de nouvelles compétences en francoprovençal pour répondre à de nouvelles exigences et pour m'adapter à de nouveaux registres, afin de le parler devant le public international d'une conférence, comme c'était le cas à Courmayeur pendant la Féta dou Patoué en 2014, ou afin de rédiger des procès-verbaux en temps réel pendant les assemblées du Conseil International du Francoprovençal, pour ne donner que deux exemples.

J'oscille entre norme et usage, entre nostalgie et vision à long terme, entre amour du pays et idéal de langue, entre des sentiments identitaires correspondant à de nombreux cercles concentriques du plus exclusif au plus inclusif et vice-versa, mais jamais je n'oublie le sentiment de liberté que me garantissent toutes ces stratégies communicatives, cette panoplie de langues avec lesquelles je jongle avec délectation. De plus en plus, car l'expérience continue. Dès que ma situation familiale me le permettra, je compte m'organiser pour aller travailler quelque temps dans une région dont je ne connais pas la langue et me remettre encore en jeu. J'ai quelques idées déjà, mais je suis trop jalouse de mes rêves pour les partager, car je sais qu'lls vont se réaliser! 